\title{
Solving the Puzzling Absolute Configuration Determination of a Flexible Molecule by Vibrational and Electronic Circular Dichroism Spectroscopies and DFT Calculations: The Case Study of a Chiral 2,2'-Dinitro-2,2'-biaziridine
}

\author{
Sergio Abbate, ${ }^{*[a, b]}$ Alessia Ciogli, ${ }^{[c]}$ Stefania Fioravanti, ${ }^{[\mathrm{d}]}$ Francesco Gasparrini, ${ }^{[\mathrm{c}]}$ \\ Giovanna Longhi, ${ }^{*[a, b]}$ Lucio Pellacani, ${ }^{[d]}$ Egon Rizzato, ${ }^{[e]}$ Domenico Spinelli, ${ }^{[e]}$ and \\ Paolo A. Tardella ${ }^{[d]}$
}

Keywords: Configuration determination / Circular dichroism / Density functional calculations / Conformation analysis / Biaziridines

The absolute configuration of a recently synthesized racemate of 2,2'-dinitro-2,2'-biaziridine (2a), a possible catalyst for asymmetric synthesis, has been determined by vibrational circular dichroism (VCD) spectroscopy in the mid-IR region and DFT calculations. Electronic circular dichroism (ECD) spectra have been obtained and Time-Dependent DFT (TDDFT) calculations have been performed and found to be in agreement with the conclusions from VCD and DFT. A detailed conformational analysis, for approximately 300 geometries, has been carried out, allowing us to find evidence for the most populated conformers chiefly contributing to VCD and ECD spectra. The proposed absolute configuration and prevalent conformers are in correspondence with an equal configuration of the nitrogen atoms of the two aziridine rings.

\section{Introduction}

Recently, by a direct aza-MIRC (Michael-initiated ring closure) reaction promoted by $\mathrm{CaO}$ on nosyloxycarbamates $\left(\mathrm{NsONHCO}_{2} \mathrm{R}^{\prime} ; \mathrm{Ns}=4-\mathrm{NO}_{2} \mathrm{C}_{6} \mathrm{H}_{4} \mathrm{SO}_{2}, \mathrm{R}^{\prime}=\mathrm{Et}, \mathrm{Bn}\right)$, the relevant ( \pm )-2,2'-dinitro-2,2' -biaziridines (2) were obtained by us $^{[1]}$ by starting from some $(E, E)$-1,4-dialkyl-2,3-dinitro1,3-butadienes (1; Scheme 1). Compounds 2, well decorated with interesting functional groups (aziridine ring, nitro, and carboxylate groups) and designed to furnish quite a few possible chemical modifications, represent versatile building blocks that are able to open the way to a variety of new compounds that could be useful as catalysts for asymmetric syntheses or as pharmacologically active substrates.

[a] Dipartimento di Scienze Biomediche e Biotecnologie, Università di Brescia,

Viale Europa 11, 25123 Brescia, Italy

Fax: +39-030-3701157

E-mail: abbate@med.unibs.it longhi@med.unibs.it

[b] CNISM,

Via della Vasca Navale 84, 00146 Roma, Italy

[c] Dipartimento di Chimica e Tecnologie del Farmaco, Università degli Studi "La Sapienza",

Piazzale A. Moro 5, 00185 Roma, Italy

[d] Dipartimento di Chimica, Università degli Studi

"La Sapienza",

Piazzale A. Moro 5, 00185 Roma, Italy

[e] Dipartimento di Chimica "G. Ciamician", Università degli Studi di Bologna,

Via Selmi 2, 40126 Bologna, Italy

Supporting information for this article is available on the WWW under http://dx.doi.org/10.1002/ejoc.201000762.

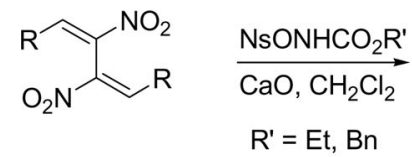

$(E, E)-1$

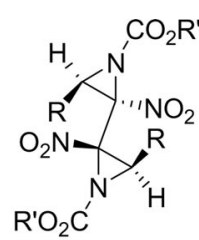

2
Scheme 1. Formation of only one racemate out of ten possible stereoisomers of $\mathbf{2}$ (one enantiomer is depicted).

Examining the structures of biaziridines 2, one can observe that they contain four stereogenic carbon atoms (stable at room temperature) that are related by symmetry in pairs. Also, aziridine nitrogen atoms are stereogenic, but in a dynamic fashion. Of course we will consider nitrogen inversion when discussing the conformational mobility. Taking into account just asymmetric carbon atoms, symmetry considerations reduce the total number of stereoisomers from the possible sixteen $\left(2^{4}\right)$ to only ten, four couples of enantiomers $\left(2 R, 2^{\prime} R, 3 R, 3^{\prime} R / 2 S, 2^{\prime} S, 3 S, 3^{\prime} S\right.$ and $2 R, 2^{\prime} R, 3 S, 3^{\prime} S / 2 S, 2^{\prime} S, 3 R, 3^{\prime} R$ with a $C_{2}$ symmetry axis, as well as $2 R, 2^{\prime} R, 3 R, 3^{\prime} S / 2 S, 2^{\prime} S, 3 S, 3^{\prime} R$ and $2 R, 2^{\prime} S, 3 R, 3^{\prime} R /$ $2 S, 2^{\prime} R, 3 S, 3^{\prime} S$ with no $C_{2}$ symmetry axis) and two meso forms $\left(2 R, 2^{\prime} S, 3 R, 3^{\prime} S=2 S, 2^{\prime} R, 3 S, 3^{\prime} R\right.$ and $2 R, 2^{\prime} S, 3 S, 3^{\prime} R$ $=2 S, 2^{\prime} R, 3 R, 3^{\prime} S$ ).

Quite unexpectedly, the HPLC analyses (carried out with UV, CD, and ELSD detectors simultaneously) of the reaction mixtures have shown that the aziridination happens to give essentially only a pair (two enantiomers) out of the ten possible stereoisomers of the 2,2'-biaziridines 2. Examina- 
tion of the ${ }^{1} \mathrm{H}$ NMR spectra of the obtained 2,2'-biaziridines has shown that the signals of the two aziridine protons are not coincident but are split apart by approximately $\delta$ $=0.45 \mathrm{ppm}$ (i.e., they are not chemically equivalent) thus allowing us to exclude the two couples containing the $C_{2}$ symmetry axis. Therefore, the obtained racemate must be one of the couples $2 R, 2^{\prime} R, 3 R, 3^{\prime} S / 2 S, 2^{\prime} S, 3 S, 3^{\prime} R$ or $2 R, 2^{\prime} S, 3 R, 3^{\prime} R / 2 S, 2^{\prime} R, 3 S, 3^{\prime} S$. Moreover, by the action of sodium iodide, $\mathbf{2} \mathbf{a}\left(\mathrm{R}=\mathrm{Me}, \mathrm{R}^{\prime}=\mathrm{Et}\right)$ gave a rearrangement with ring enlargement furnishing the relevant 5,5'-dimethyl4,4',5,5'-tetrahydro-3,3'-bi(1,2,4-oxadiazole) 2,2'-dioxides (3a) ${ }^{[2]}$ still as a single racemate through an $\mathrm{S}_{\mathrm{N}} 2-\mathrm{S}_{\mathrm{N}} 2$ domino ring-opening/ring-closure reaction with complete stereoselectivity $^{[1]}$ (Scheme 2).

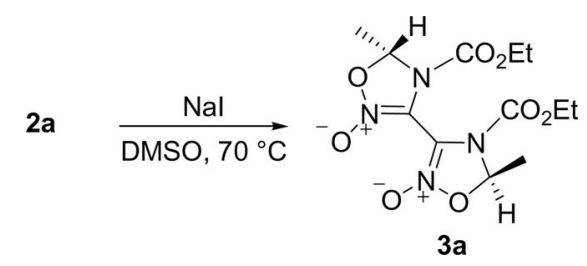

Scheme 2. Ring expansion of $\mathbf{2 a}$ to $\mathbf{3 a}$ (only one enantiomer is depicted for 3a).

This last result allows us to deduce that the two surviving chiral centers must be homochiral (the heterochiral combination should give rise to a meso-oxadiazolidine) and that the major isolated couple of $\mathbf{2 a}$ must be the racemate $2 R, 2^{\prime} S, 3 R, 3^{\prime} R / 2 S, 2^{\prime} R, 3 S, 3^{\prime} S$ (Figure 1).

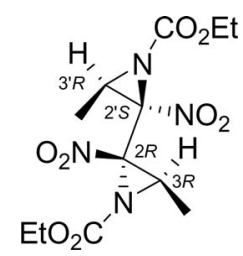

Figure 1. Structure of $\left(2 R, 2^{\prime} S, 3 R, 3^{\prime} R\right)-\mathbf{2 a}$

The problem of configurational assignment of the two eluted enantiomers, $2 R, 2^{\prime} S, 3 R, 3^{\prime} R$ versus $2 S, 2^{\prime} R, 3 S, 3^{\prime} S$, of racemate $\mathbf{2} \mathbf{a}$ is solved by first measuring their chiroptical properties and comparing them to the corresponding calculated data on a chosen configuration. The advantage of using the vibrational circular dichroism (VCD) technique is in the fact that usually many bands with different signs are observed in the mid-IR region, and reliable VCD spectra calculations can be performed, as documented by well-settled literature relevant for this work. ${ }^{[3,4]}$ For this reason, a good correspondence between the observed spectra and the calculated ones provides unambiguous absolute configuration (AC) assignment. Of course other chiroptical properties may give and will give, as shown later, further confidence to the assignment based on VCD.

\section{Results and Discussion}

To deepen the results of ref. ${ }^{[1]}$, in this work we will determine the $\mathrm{AC}$ of the two enantiomers of $\mathbf{2} \mathbf{a}$ chosen as model compounds. The first requirement, to carry out this project, was the availability of the pure enantiomers constituting the racemate $\mathbf{2} \mathbf{a}$, by starting from a crude sample. The enantioseparation was successfully obtained on a covalently bonded polysaccharide-based stationary phase (Chiralpak IA) with an enantiomeric excess of approximately $97 \%$ for both enantiomers. The first eluted enantiomer, named (-)2a-I gives $[\alpha]_{\mathrm{D}}^{25}=-174.76\left(c=0.26 \mathrm{w} / \mathrm{v}-\%, \mathrm{CHCl}_{3}\right)$ and the on-line CD-spectrum shows a negative $\mathrm{CD}$ band close to $275 \mathrm{~nm}$. The second eluted enantiomer, (+)-2a-II, gives $[a]_{\mathrm{D}}^{25}=+167.19\left(c=0.26 \mathrm{w} / \mathrm{v}-\%, \mathrm{CHCl}_{3}\right)$ and a positive CD band at about $275 \mathrm{~nm}$ (Figure 2).

Since enantioselective chromatography and previously determined chiroptical properties $\left([\alpha]_{\mathrm{D}}\right.$ and on-line ECD) do not define the AC of enantiomers of $\mathbf{2 a}$, we have determined their ACs by using chiroptical spectroscopy.

The measured VCD (Figure S1 of the Supporting Information) and electronic circular dichroism spectra (ECD; Figure S2 of the Supporting Information) for both enantiomers, (-)-2a-I and (+)-2a-II, have been used to elucidate their $A C^{[5-9]}$ by DFT calculations. Many papers presented, through the years, very convincing and consistent examples, whereby VCD, in conjunction with DFT calculations, allows one to go beyond the ambiguities of ECD spectroscopy, when the results from the latter technique are analyzed with an insufficient level of theory. ${ }^{[10]}$ Nowadays, time-dependent DFT (TD-DFT) has, most of the time, allowed the correct interpretation of the ECD spectra. ${ }^{[11-12]}$ However, VCD represents the most powerful technique for the goal of AC elucidation and was even demonstrated to be superior to X-ray diffraction data in a few instances. ${ }^{[13]}$

To establish AC, we have adopted the $2 R, 2^{\prime} S, 3 R, 3^{\prime} R$ structure for our calculations. We report the schematics and a three-dimensional structure of the $\left(2 R, 2^{\prime} S, 3 R, 3^{\prime} R\right)$-aziridine enantiomer in Figure 3, for which we have evidenced the definition of the most important conformational degrees of freedom and have presented the atomic numbering used throughout this paper. Indeed the major task is the conformational study of this molecule: VCD and absorption spectra are to be calculated on the optimized conformational structures. For conformational analysis, we have to consider all possible allowed values for torsions relative to the dihedral angles illustrated in Figure 3.

As a preliminary step, we have focused just on the central part of the molecule (Figure 4) to study the hindered rotation defined by the dihedral angle $\delta$ (N1-C2-C12-N14). With a scan at the B3LYP/6-31+G** level in Gaussian $03,{ }^{[14]}$ we obtain just two minima close to what we may call gauche $(-)$ and gauche $(+)$ conformers (corresponding to the angles $\delta=-65$ and $+70^{\circ}$, respectively), the geometry corresponding to the trans position, which is associated with a flat maximum, the cis one instead exhibiting a very steep and high barrier.

Considering now the complete molecule (Figure 3), we assume that the two dihedral angles corresponding to the single $\mathrm{C}-\mathrm{O}$ bonds, $\mathrm{O}=\mathrm{C}-\mathrm{O}-\mathrm{Et}$ (C21-O17-C15-O16 and C24-O20-C18-O19), are in a cis position. This is usually the preferred conformation of such groups and, in this par- 

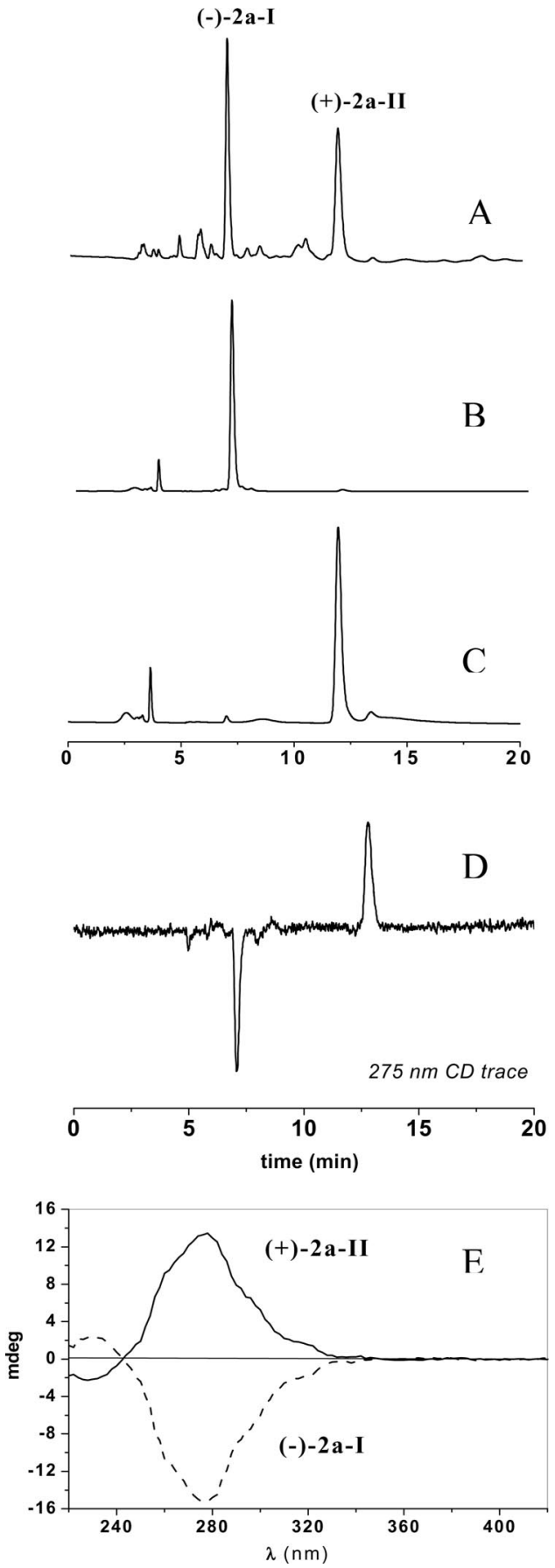

Figure 2. Enantioseparation of 2a. UV $220 \mathrm{~nm}$ chromatographic traces of crude racemic 2a (A). Purity of first (B) and second (C) collected enantiomers by semi-preparative HPLC. CD $275 \mathrm{~nm}$ chromatographic trace of crude racemic 2a (D). On-line ECD spectra of both enantiomers (E).

ticular situation, the alternative trans conformation is forbidden by steric hindrance between the ethyl groups and the adjacent ring. With these considerations in mind, we optimized the structure of the whole molecule at the AM1 level, by starting from initial conditions defined by the systematic combination of the values reported below for the following dihedral angles:
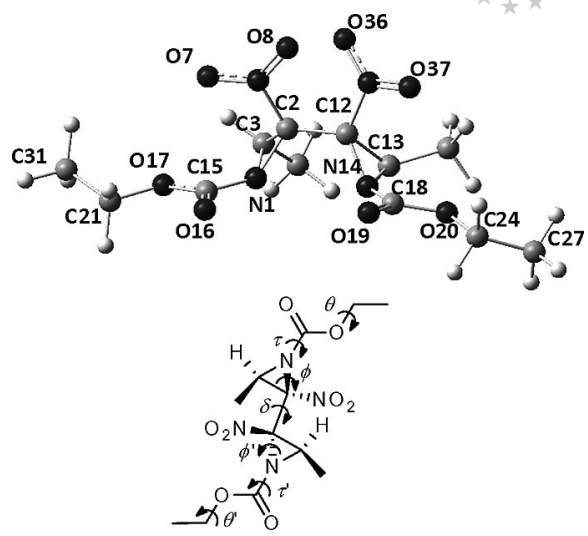

Figure 3. Atomic numbering for heavier atoms mainly involved in the conformational analysis (top). Definition of the relevant dihedral angles for the $2 R, 2^{\prime} S, 3 R, 3^{\prime} R$ enantiomer of $\mathbf{2 a}$ pointing out the most important conformational degrees of freedom (angles: $\delta$, $\tau, \tau^{\prime}, \theta, \theta^{\prime}, \phi, \phi^{\prime} ;$ bottom).

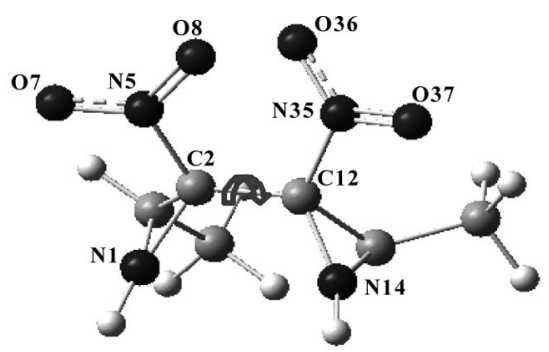

$\mathrm{kcal} / \mathrm{mol}$

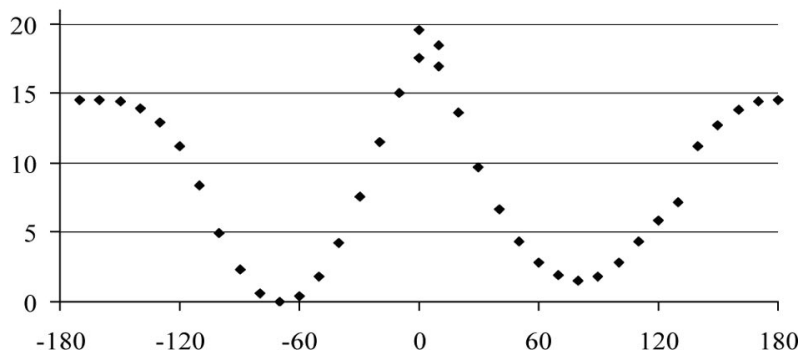

Figure 4. Potential-energy curve (PEC) of the $2 R, 2^{\prime} S, 3 R, 3^{\prime} R$ enantiomer of a model molecule without $\mathrm{CO}_{2} \mathrm{Et}$ groups for rotation of the dihedral angle $\delta(\mathrm{N} 1-\mathrm{C} 2-\mathrm{C} 12-\mathrm{N} 14)$ around the central C2$\mathrm{C} 12$ bond. The PEC is obtained on the basis of DFT calculations at the $\mathrm{B} 3 \mathrm{LYP} / 6-31+\mathrm{G}^{* *}$ level, the remaining degrees of freedom having been optimized (see text).

1) The dihedral angle $\delta$, at the gauche(+) and gauche(-) positions.

2) The angles $\tau$ and $\tau^{\prime}(\mathrm{C} 2-\mathrm{N} 1-\mathrm{C} 15-\mathrm{O} 16$ and $\mathrm{C} 12-\mathrm{N} 14$ C18-O19), each one possessing pairs of values differing roughly by $180^{\circ}$.

3) The angles $\theta$ and $\theta^{\prime}(\mathrm{C} 31-\mathrm{C} 21-\mathrm{O} 17-\mathrm{C} 15$ and $\mathrm{C} 27-$ $\mathrm{C} 24-\mathrm{O} 20-\mathrm{C} 18)$, each one at the trans, gauche $(+)$ and gauche (-) positions.

4) Finally the angles $\phi$ and $\phi^{\prime}(\mathrm{C} 15-\mathrm{N} 1-\mathrm{C} 2-\mathrm{C} 12$ and $\mathrm{C} 2-$ $\mathrm{C} 12-\mathrm{N} 14-\mathrm{C} 18)$ for values of about $130^{\circ}$ corresponding to an orientation for the $\mathrm{CO}_{2} \mathrm{Et}$ group relative to the aziridinic ring plane that we may define as $u p(u)$, and of about $0^{\circ}$ (cis) corresponding to an orientation for the same group 
that we define down (d). These two values of $\phi$ and $\phi^{\prime}$ are related to the configuration of the two aziridinic nitrogen atoms: the up position for $\phi$ corresponds to the $S$ configuration of nitrogen 1 (Figure 3 ) and the up position of $\phi^{\prime}$ corresponds to the $R$ configuration of nitrogen 14 . Since the inversion of nitrogen atoms strongly depends energetically on the other conformational degrees of freedom, at this point, we will consider the angles $\phi$ and $\phi^{\prime}$ in the conformational analysis: the final result will define the absolute configuration of the two nitrogen atoms.

All these possibilities together amount to 288 initial geometries that need to be tested. However, outside this set, we had also to consider another 36 initial conformations for values of $\delta$ larger than $100^{\circ}$, namely beyond the second minimum of Figure 4, for $\phi$ values corresponding to the $u p$ position, whereas $\phi^{\prime}$ is in the down position, with the two usual possible values for $\tau$ and $\tau^{\prime}$ and the three possible values for $\theta$ and $\theta^{\prime}$, as above.

The whole 324 initial geometries converged to 286 distinct conformers at the AM1 level, as reported in Table S1 of the Supporting Information, numbered in order of increasing energy. The conformers are labeled by evidencing the gross characteristics of the principal dihedral angles, namely the + or - sign for $\delta, u=u p, d=$ down for both $\phi$ and $\phi^{\prime}$, a capital letter ranging from $\mathrm{A}$ through $\mathrm{P}$ assigned to possible combinations of $\tau$ and $\tau^{\prime}$ values, whereas for $\theta$ and $\theta^{\prime}$, we do not need to have any particular label, as will become clear further on.

From the calculations carried out at the AM1 level, the most probable conformations appear to be the ones with $\phi$ and $\phi^{\prime}$ up. We optimized the lower-energy conformers' geometries at B3LYP/6-31+G** level and we calculated VCD and absorption spectra within the harmonic approximation. ${ }^{[15,16]}$ For a few cases, we checked that a change in just $\theta$ and $\theta^{\prime}$ has little influence on the shape of the VCD spectrum. A comparison is shown in Figure S3 of the Supporting Information. In Figure S4 of the Supporting Information we show the VCD spectra for a first set of eight conformers with $\phi$ and $\phi^{\prime}$ in the $u p$ position, with the two possible + and - values for $\delta$, and for the four combinations A-D of two possible values for each angle $\tau$ and $\tau^{\prime}$ : the eight conformations are indicated by the four-symbol notation introduced above, namely $-u u \mathrm{~A},-u u \mathrm{~B},-u u \mathrm{C},-u u \mathrm{D}$, $+u u \mathrm{~A},+u u \mathrm{~B},+u u \mathrm{C}$, and $+u u \mathrm{D}$.

The results obtained by considering the conformers with a low AM1 energy are unsatisfactory, especially with regard to the simultaneous match of the sign of the two VCD couplets centered at about 1765 and $1250 \mathrm{~cm}^{-1}$; for this reason, we were forced to consider other conformations besides the ones discussed above, even if they exhibited high energy values in the semi-empirical analysis. In this case, AM1 calculations do not adequately represent the relative energies, especially for the conformers differing in the $\phi$ and $\phi^{\prime}$ angles. Following this hypothesis, we have examined the other cases: $\phi=$ down and $\phi^{\prime}=u p, \phi=u p$ and $\phi^{\prime}=$ down, and finally $\phi=$ down and $\phi^{\prime}=$ down, obviously still considering the different possibilities for $\delta, \tau$, and $\tau^{\prime}$. To conclude, we took into account the lowest AM1 energy conformers in each homologous $\delta, \phi, \phi^{\prime}, \tau, \tau^{\prime}$ subset and optimized them at the B3LYP/6-31+G** level. To obtain the correct population values, for the most probable cases thus obtained, we had to consider also the different possible values for $\theta$ and $\theta^{\prime}$ at the same level. All these tested examples are reported in Table S2 of the Supporting Information with the numbering adopted from AM1 results given in the first column. It is evident that the preferred conformations have $\phi$ $=u p$ and $\phi^{\prime}=$ down.

Despite the large number of possible conformations, just a few conformers are populated (if one disregards ethyl rotations, i.e. angles $\theta$ and $\theta^{\prime}$ ); all of them have a $\delta$ value close to $-60^{\circ}$, with $\phi$ always $u p$. The different conformational behavior with respect to angle $\phi$ and $\phi^{\prime}$ is due to the different chirality of the asymmetric carbon atoms $\mathrm{C} 3$ and $\mathrm{C} 13: \phi^{\prime}$ down lowers the steric hindrance between the ring methyl and the $\mathrm{CO}_{2}$ Et group. In Figure 5, the three most populated

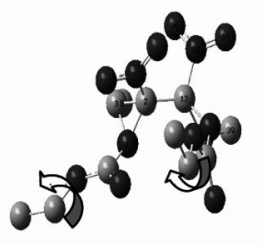

$-u d \mathrm{H}$

$63 \%$ with six

populated conformers

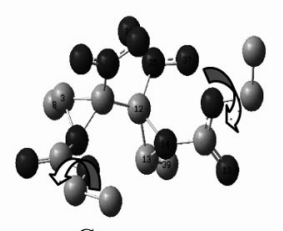

$-u u \mathrm{C}$

$17 \%$ with nine populated conformers

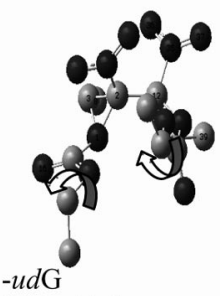

$13 \%$ with five populated conformers
Figure 5. Representation of the three most populated conformers: $-u d \mathrm{H}(63 \%),-u u \mathrm{C}(17 \%)$, and $-u d \mathrm{G}(13 \%)$ as obtained on the basis of DFT calculations at the B3LYP/6-31+G** level. The population factors are summed over all possible conformations of the $\mathrm{CO}_{2}$ Et groups ( $\mathrm{H}$ atoms are not reported).

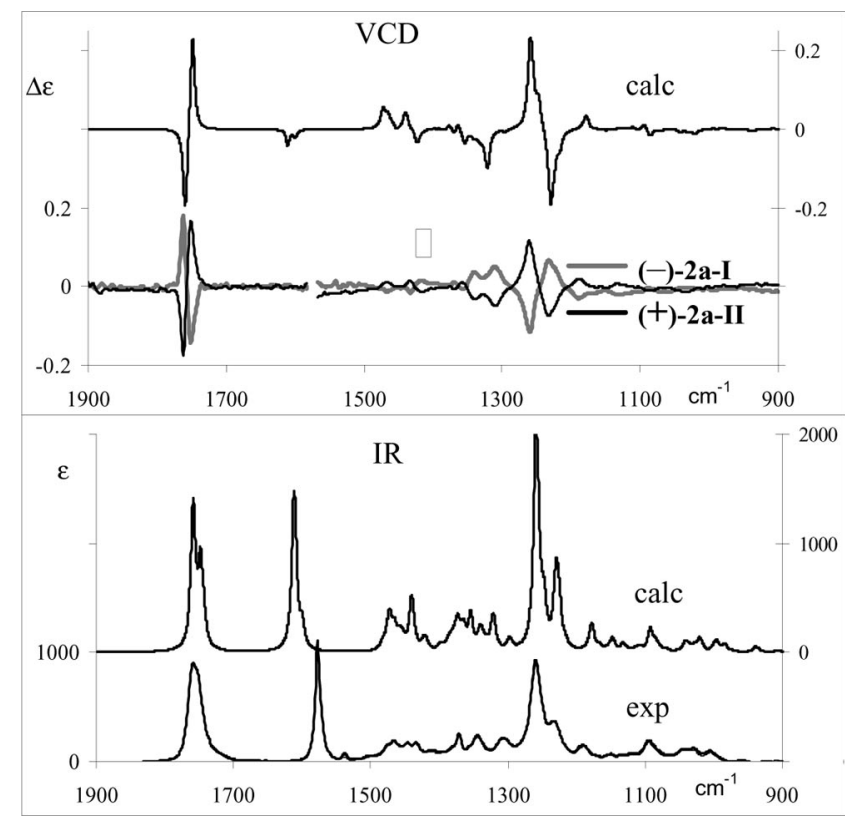

Figure 6. Comparison of the (-)-2a-I and (+)-2a-II experimental data (lower traces) with the Boltzmann weighted average calculated spectra of the $2 R, 2^{\prime} S, 3 R, 3^{\prime} R$ enantiomer (calculated frequencies are scaled by a factor of 0.975 ). 
conformers are reported with their population factors obtained by summing up over all the contributions due to different $\theta$ and $\theta^{\prime}$ values. For the most probable conformers of Table S2 of the Supporting Information, we observe that the two carboxylic groups avoid being mutually parallel and the four possible combinations of values for $\tau$ and $\tau^{\prime}$, once fixed $\delta, \phi$, and $\phi^{\prime}$, correspond to exchanging the position of the two oxygen atoms of the carboxylic group (the conformation of the two carboxylic groups is indicated with the capital letter introduced above).

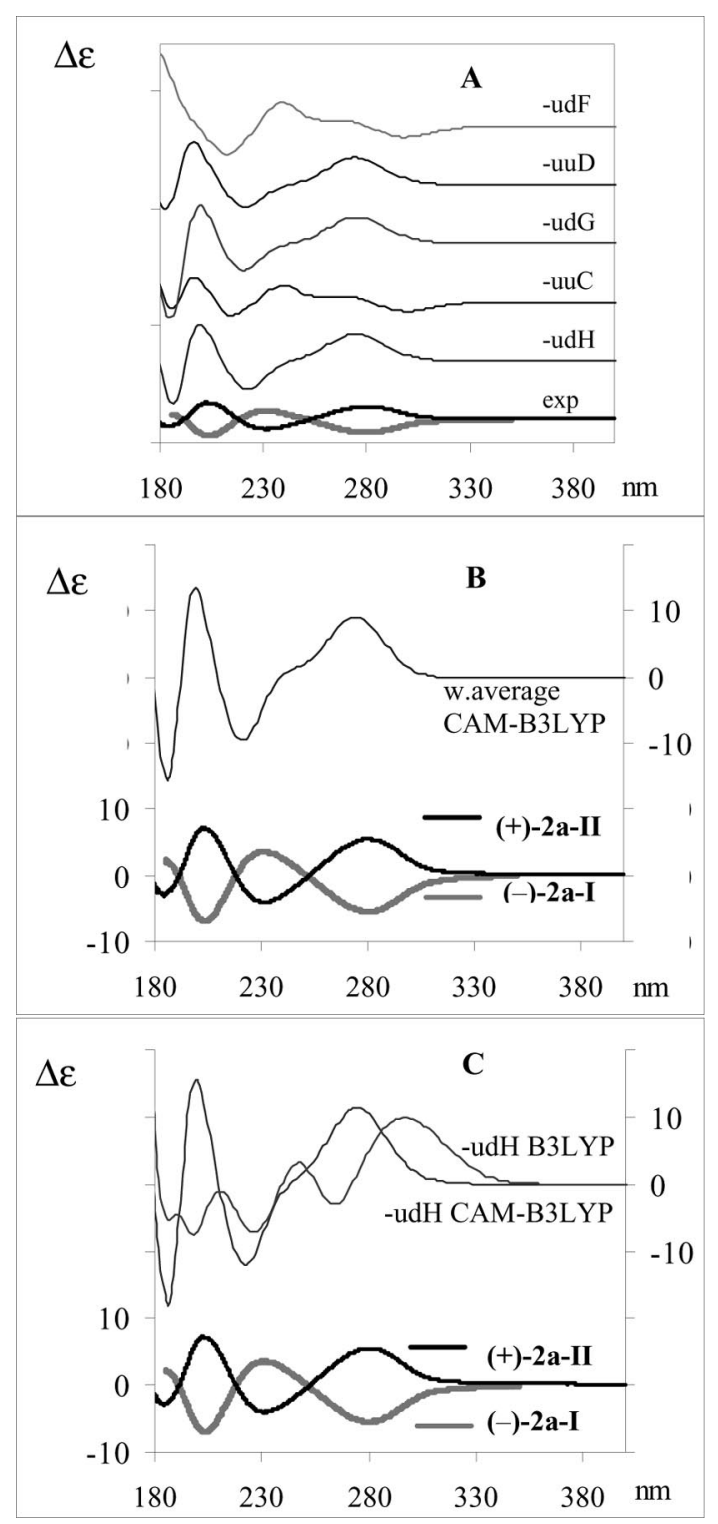

Figure 7. A) Comparison of experimental (lowest traces) with calculated (upper traces) ECD spectra of (-)-2a-I and (+)-2a-II for the five most populated conformers of the $2 R, 2^{\prime} S, 3 R, 3^{\prime} R$ enantiomer, based on DFT calculations at the CAMB3LYP/6-31+G** level. B) Comparison of experimental (lower traces) with CAMB3LYP/6$31+\mathrm{G}^{* *}$ level calculated (upper traces) ECD spectra for the conformational average. C) Comparison of experimental (lower traces) with calculated (upper traces) ECD spectra for the most populated conformer $(-u d \mathrm{H})$ obtained on the basis of DFT calculations at the B3LYP/6-31+G** level and at the CAMB3LYP/6-31+G** level.
We report in Figure S5 of the Supporting Information the VCD and absorption spectra of the most populated conformers (corresponding to a $93 \%$ population within the subset of 70 cases reported in Table S2 of the Supporting Information). In Figure 6, finally we compare the experimental data with the Boltzmann-weighted average calculated spectra, after scaling the calculated frequencies by a 0.975 factor for ease of comparison. A quite good correspondence is obtained: this allows us to establish unequivocally that the second eluted compound (+)-2a-II has a $2 R, 2^{\prime} S, 3 R, 3^{\prime} R$ configuration. The fact that we obtain a good match of experimental and calculated spectra means also that the conformational analysis conducted here gives a good representation of the molecular system in $\mathrm{CCl}_{4}$ solution, that is, the obtained population factors are reliable; in particular, the conformation here called $-u d \mathrm{H}$ (allowing for complete mobility of end-ethyls) is populated at $63 \%$, and shows already almost the correct VCD spectrum. Two other probable conformers are $-u u \mathrm{C}$ and $-u d \mathrm{G}$, which are populated at 17 and $13 \%$, respectively.

The assignment is also confirmed by the calculations of ECD spectra based on the TD-DFT method, ${ }^{[11,12]}$ which are presented in Figure 7. Also in this case, the conformation of the terminal ethyl groups does not influence the shape of the spectra at the wavelengths of interest. We notice that the B3LYP functional does not perfectly account for the observed data, even though the assignment is still correct. It is known that the Coulomb-attenuated CAMB3LYP functional[17,18] gives better results, especially for $\mathrm{N}$ containing molecules. ${ }^{[19]}$ A comparison of the performance of the two functionals on the most populated conformer is given in part $\mathrm{C}$ of Figure 7. The calculated average spectrum at the CAM-B3LYP level is in good correspondence with experimental observations.

\section{Conclusions}

We reiterate that this work is strictly linked to our previous work: ${ }^{[1]}$ by allowing the complete definition of the major pairs of enantiomers among the possible ones resulting from the reaction of Scheme 1. This work may provide information concerning future work on catalysts for asymmetric syntheses. In addition, by definitively confirming the structure of the isolated racemate, this work can also help the elucidation of the reaction mechanism, with respect to the generation and control of the four "stable" chiral centers involved in the bisaziridination process, which in turn may give rise to multiple conformations that need to be examined.

DFT calculations in vacuo are usually accepted as well suited to represent molecules in apolar solvents, such as $\mathrm{CCl}_{4}$. For this reason, after careful investigation of the possible values of important dihedral angles, one can adopt the population factors obtained on the basis of free-energy calculations in vacuo. This analysis shows that just a few conformers are important, and due to the fact that the quite large signals recorded in VCD spectra are nearly unper- 
turbed by conformations of end-ethyls, we can state with confidence that the absolute configuration of the (+)-2a-II eluted biaziridine previously described by $\mathrm{us}^{[1]}$ is $2 R, 2^{\prime} S, 3 R, 3^{\prime} R$. The study of this molecule has once more evidenced the inadequacy of semi-empirical calculations for even preliminary conformational screening, if adopted without a careful check. The number of possible conformers being high, one should consider the conformational analysis, even conducted at the DFT level, to be reliable only if supported by experimental data. For this reason, we think that it is quite important to be able to reproduce the VCD spectrum, which possesses many independent data, as well as the ECD spectrum, as conducted in the present work.

Coming finally to comment on the predominant conformation for the assigned configuration, namely the one we called $-u d \mathrm{H}$, we first observe that the negative central dihedral angle $\delta$ is predicted even at the semi-empirical level (see Table S1 of the Supporting Information). The succession $u d$ attributed to $\phi$ and $\phi^{\prime}$, respectively, is also quite important: as previously noted, we observe that the conformational preference $u d$ assigns the same $S$ configuration to the $\mathrm{N}$ atoms of the two aziridine rings, if the lone pair is included in the definition of the $\mathrm{AC}$ of the $\mathrm{N}$ atom. Earlier in the literature, ${ }^{[20]} \mathrm{VCD}$ already succeeded to define the $\mathrm{AC}$ of $\mathrm{N}$ atoms or, as defined in ref. ${ }^{[20]}$, to choose between "invertomers". The conformational preference denoted as $\mathrm{H}$ is related to the dihedral angles $\tau$ and $\tau^{\prime}$, the first one with values at approximately $-60^{\circ}$ and the second one at approximately $+120^{\circ}$. The ensemble of the three conformational characteristics $u d \mathrm{H}$ is seen (see Figure S5 in the Supporting Information) to be related to the correct prediction of the VCD doublet at approximately $1750 \mathrm{~cm}^{-1}$; indeed, they ensure the correct relative position of the $\mathrm{C}=\mathrm{O}$ bonds of the two carboxylic groups. Regarding the VCD doublet at approximately $1250 \mathrm{~cm}^{-1}$, almost all calculated VCD spectra of Figure S5 of the Supporting Information are correct in that respect; the wider bandwidth observed for this doublet is due to the differences in calculated frequencies of the doublet depending on conformation. Finally, the relative position of the two nitro groups is mostly determined by the negative $\delta$ angle, the stretching vibrations of these groups are responsible of the sharp intense absorption band at $1590 \mathrm{~cm}^{-1}$.

\section{Experimental Section}

General: Full details concerning materials (2a, 3a, $\mathrm{CH}_{2} \mathrm{Cl}_{2}, \mathrm{CHCl}_{3}$, $\mathrm{CCl}_{4}$, and $\mathrm{MeCN}$ ), separation of 2a by chiral chromatography, determination of polarimetric, mid-IR VCD, and ECD measurements, as well as DFT and TD-DFT calculations for the $2 R, 2^{\prime} S, 3 R, 3^{\prime} R$ enantiomer of $\mathbf{2 a}$ are reported in the Supporting Information.

Superimposed experimental VCD spectra of (-)-2a-I and (+)-2a-II and superimposed experimental ECD spectra of (-)-2a-I and (+)2a-II are reported in Figures S1 and S2, respectively (Supporting Information).
Energy values and geometrical characteristics of the conformers obtained with AM1 optimization are reported in Table S1 (Supporting Information). Energy values and geometrical characteristics of the conformers obtained with B3LYP/6-31+G** optimization are reported in Table S2 (Supporting Information). Comparison of VCD spectra on two groups of conformers discussed in the text to analyze the dependence of VCD spectra on the different dihedral angles are reported in Figures S3 and S4 (Supporting Information). In Figure S5 (Supporting Information) full comparison of calculated IR and VCD spectra for the first most populated 20 conformers of $\left(2 R, 2^{\prime} S, 3 R, 3^{\prime} R\right)$-biaziridine with the superimposed experimental spectra of $(-)-\mathbf{2} \mathbf{a}-\mathbf{I}$ and $(+)-\mathbf{2} \mathbf{a}-\mathbf{I I}$ is given. This material is available free of charge via Internet at http://www.chemeurj.org.

Supporting Information (see also the footnote on the first page of this article): In this section the complete description of experimental procedures and DFT calculations with references are listed.

\section{Acknowledgments}

The Italian Ministero dell'Università e della Ricerca (MIUR) and Università degli Studi di Roma "La Sapienza" are gratefully acknowledged for financial support (PRIN 2007FJC4SF_005, PRIN 20078J9L2A_005, PRIN, 2008LYSEBR_003). The Consorzio Interuniversitario Lombardo per l'Elaborazione Automatica (CILEA), via R. Sanzio 4, 20090 Segrate (MI), Italy, is acknowledged for kindly allowing the use of computational facilities.

[1] A. Ciogli, S. Fioravanti, F. Gasparrini, L. Pellacani, E. Rizzato, D. Spinelli, P. A. Tardella, J. Org. Chem. 2009, 74, 9314-9318.

[2] Several 1,2,4-oxadiazole derivatives, which can be considered hydrolysis-resistant bioisosters of esters or amides, have been suggested and/or used as antitussive agents, coronary vasodilators, local anaesthetic, antispasmodic, antiinflammatory and analgesic agents, muscarinic agonists and antagonists, depressant of CNS, etc. (A. R. Katritzky, C. A. Ramsdem, E. F. V. Scriven, R. J. K. Tylot (Eds.), Comprehensive Heterocyclic Chemistry III, vol. 5, Elsevier, London, UK). Very recently, some derivatives have shown interesting properties as inhibitors of MDR1 activity (M. Viale, C. Cordazzo, B. Cosimelli, D. de Totero, P. Castagnola, C. Aiello, E. Severi, G. Petrillo, M. Cianfriglia, D. Spinelli, J. Med. Chem. 2009, 52, 259-266).

[3] P. J. Stephens, F. J. Devlin, F. Gasparrini, A. Ciogli, D. Spinelli, B. Cosimelli, J. Org. Chem. 2007, 72, 4707-4715.

[4] P. M. Wood, L. W. L. Woo, J. R. Labrosse, M. N. Trusselle, S. Abbate, G. Longhi, E. Castiglioni, F. Lebon, A. Purohit, M. J. Reed, B. V. L. Potter, J. Med. Chem. 2008, 51, 4226- 4238.

[5] P. J. Stephens, F. J. Devlin, J. J. Pan, Chirality 2008, 20, 643663.

[6] L. A. Nafie, T. B. Freedman, Vibrational Optical Activity Theory, in: Circular Dichroism: Principles and Applications, 2nd ed. (Eds.: N. Berova, K. Nakanishi, R. W. Woody), WileyVCH, New York, 2000, chapter 4, pp. 97-132.

[7] P. L. Polavarapu, J. He, Anal. Chem. A 2004, 76, 61A-67A.

[8] T. Kuppens, P. Bultinck, A. Langeneker, Drug Discovery Today: Technologies 2004, 269-275.

[9] G. Longhi, S. Abbate, R. Gangemi, E. Giorgio, C. Rosini, J. Phys. Chem. A 2006, 110, 4958-4968.

[10] A. Aamouche, F. J. Devlin, P. J. Stephens, J. Am. Chem. Soc. 2000, 122, 2346-2354.

[11] C. Diedrich, S. Grimme, J. Phys. Chem. A 2003, 107, 2524 2539.

[12] A. E. Hansen, T. D. Bouman, Adv. Chem. Phys. 1980, 44, 454 644.

[13] P. J. Stephens, F. J. Devlin, P. Besse, Tetrahedron: Asymmetry 2005, 16, 1557-1566. 
[14] M. J. Frisch, G. W. Trucks, H. B. Schlegel, G. E. Scuseria, M. A. Robb, J. R. Cheeseman, J. A. Montgomery Jr., T. Vreven, K. N. Kudin, J. C. Burant, J. M. Millam, S. S. Iyengar, J. Tomasi, V. Barone, B. Mennucci, M. Cossi, G. Scalmani, N. Rega, G. A. Petersson, H. Nakatsuji, M. Hada, M. Ehara, K. Toyota, R. Fukuda, J. Hasegawa, M. Ishida, T. Nakajima, Y. Honda, O. Kitao, H. Nakai, M. Klene, X. Li, J. E. Knox, H. P. Hratchian, J. B. Cross, V. Bakken, C. Adamo, J. Jaramillo, R. Gomperts, R. E. Stratmann, O. Yazyev, A. J. Austin, R. Cammi, C. Pomelli, J. W. Ochterski, P. Y. Ayala, K. Morokuma, G. A. Voth, P. Salvador, J. J. Dannenberg, V. G. Zakrzewski, S. Dapprich, A. D. Daniels, M. C. Strain, O. Farkas, D. K. Malick, A. D. Rabuck, K. Raghavachari, J. B. Foresman, J. V. Ortiz, Q. Cui, A. G. Baboul, S. Clifford, J. Cioslowski, B. B. Stefanov, G. Liu, A. Liashenko, P. Piskorz, I. Komaromi, R. L. Martin, D. J. Fox, T. Keith, M. A. Al-Laham, C. Y. Peng, A. Nanayakkara, M. Challacombe, P. M. W. Gill, B. Johnson, W. Chen, M. W. Wong, C. Gonzalez, J. A. Pople, Gaussian 03, rev. B.05, Gaussian, Inc., Pittsburgh, PA, 2003.

[15] P. J. Stephens, J. Phys. Chem. 1985, 89, 748-752.

[16] a) P. J. Stephens, M. A. Lowe, Ann. Rev. Phys. Chem. 1985, 36, 213-241; b) P. J. Stephens, C. S. Ashvar, F. J. Devlin, J. R. Cheeseman, M. J. Frisch, Mol. Phys. 1996, 89, 579-594.

[17] T. Yanai, D. P. Tew, N. C. Handy, Chem. Phys. Lett. 2004, 393, 51-57.
[18] M. J. Frisch, G. W. Trucks, H. B. Schlegel, G. E. Scuseria, M. A. Robb, J. R. Cheeseman, J. A. Montgomery Jr., T. Vreven, K. N. Kudin, J. C. Burant, J. M. Millam, S. S. Iyengar, J. Tomasi, V. Barone, B. Mennucci, M. Cossi, G. Scalmani, N. Rega, G. A. Petersson, H. Nakatsuji, M. Hada, M. Ehara, K. Toyota, R. Fukuda, J. Hasegawa, M. Ishida, T. Nakajima, Y. Honda, O. Kitao, H. Nakai, M. Klene, X. Li, J. E. Knox, H. P. Hratchian, J. B. Cross, V. Bakken, C. Adamo, J. Jaramillo, R. Gomperts, R. E. Stratmann, O. Yazyev, A. J. Austin, R. Cammi, C. Pomelli, J. W. Ochterski, P. Y. Ayala, K. Morokuma, G. A. Voth, P. Salvador, J. J. Dannenberg, V. G. Zakrzewski, S. Dapprich, A. D. Daniels, M. C. Strain, O. Farkas, D. K. Malick, A. D. Rabuck, K. Raghavachari, J. B. Foresman, J. V. Ortiz, Q. Cui, A. G. Baboul, S. Clifford, J. Cioslowski, B. B. Stefanov, G. Liu, A. Liashenko, P. Piskorz, I. Komaromi, R. L. Martin, D. J. Fox, T. Keith, M. A. Al-Laham, C. Y. Peng, A. Nanayakkara, M. Challacombe, P. M. W. Gill, B. Johnson, W. Chen, M. W. Wong, C. Gonzalez, J. A. Pople, Gaussian 09, Gaussian, Inc., Pittsburgh, PA, 2009.

[19] M. J. G. Peach, C. R. Le Sueur, K. Ruud, M. Guillaume, D. J. Tozer, Phys. Chem. Chem. Phys. 2009, 11, 4465-4470.

[20] A. Rauk, T. Eggimann, H. Wieser, G. V. Shustov, D. Y. Yang, Can. J. Chem. 1994, 72, 506-513.

Received: May 27, 2010

Published Online: September 23, 2010 